\title{
Development of virtual reality support to factory layout planning
}

\author{
Liang Gong $^{1}$ (D) Jonatan Berglund ${ }^{1} \cdot$ Åsa Fast-Berglund ${ }^{1} \cdot$ Björn Johansson ${ }^{1} \cdot$ Zhiping Wang $^{2} \cdot$ Tobias Börjesson $^{3}$
}

Received: 11 October 2018 / Accepted: 8 January 2019 / Published online: 15 February 2019

(c) The Author(s) 2019

\begin{abstract}
Virtual reality (VR) technology has become ever mature today with affordable and yet powerful hardware. In the manufacturing industry, there is a growing interest of adopting VR to improve existing work procedures. Factory layout planning (FLP) is a long standing area in production engineering that sees great potentials of VR integration. Virtual reality supported layout planning (VLP) is gaining wider attention in research and practice as the virtual environment allows designers to test out "what if" scenarios in relative ease. However, previous research of VLP mostly focus on general layout planning but not the detailed level planning. Also, it is reported that the virtual modeling process is time-consuming and costly. In this study, we propose a point cloud based virtual factory modelling approach for the VLP tasks. It incorporates point cloud representation of physical environment with CAD data to model the virtual factory with the aims of simplifying the modelling process and improving decision-making for the VLP tasks. The proposed approach is exemplified and refined through three industrial cases. The implementations and results of the cases are highlighted and discussed in details. At the end, a general guidance for VLP is extracted and presented for future point cloud based VR support in FLP tasks.
\end{abstract}

Keywords Virtual reality $\cdot 3 \mathrm{D}$ laser scanning $\cdot$ Point cloud $\cdot$ Factory layout planning

\section{Introduction}

Virtual reality (VR) technology has become ever mature today with affordable and yet powerful hardware. In the manufacturing industry, there is a growing interest of adopting VR to improve existing work procedures. Factory layout planning (FLP) is considered as one of the most important aspects to the success of a manufacturing company. Previous studies indicate a well-designed manufacturing layout can reduce the operating cost by 50\% [1]. With the increasing complexity of the manufactured products and the demands for higher efficiency, various research and practice have been devoted to resolve it. Virtual reality supported layout planning (VLP) is gaining wider attention in research and practice as the virtual environment allows designers to test out "what if" scenarios in relative ease. Muther is one of the first to propose the systematic approach that takes the whole production process into account and considers the layout plan process as

Liang Gong

liang.gong@chalmers.se

Chalmers tekniska hogskola, Göteborg, Sweden

2 Volvo Group Trucks, Göteborg, Sweden

3 Swedish Match AB, Göteborg, Sweden a loop [2]. Since then many tools and methods have emerged to cope with the ever challenging task.

Among the existing approaches, most of them mainly focus on the quantitative measures such as travel distance, time, frequency, throughout, by applying mathematical models and algorithms to select the optimal solution [3,4]. It is proved to be efficient for general layout planning, but it is less satisfactory for the detailed layout planning when factors such as safety, ergonomics and operator preference are need to be considered [5]. Additionally, many of previous studies assume the tasks to be carried out in an open space as new layout planning [6]. While existing factories require constant changes and upgrade of the layouts so that it can adapt to new requirements such as changed product design, safety, ergonomics, etc. In the last decade, with the advancement of the virtual technologies, virtual layout plan (VLP) has drawn much attention because of its advantages in the richness and flexibility in the computerized virtual environment. It is believed that the visualization and interaction provided in the virtual model would help bringing stakeholders closer to the decision making process and thus improve the quality in both qualitative and quantitative perspectives [7]. The main challenge identified from previous virtual layout planning approaches are the time consuming process of virtual 
modeling. This is especially true when redesigning existing factories, where the detail environment are usually too complex to model precisely in CAD software.

In this study, we propose a point cloud based virtual factory modelling approach for the VLP tasks. It incorporates point cloud with CAD data to model the virtual factory with the following aims:

1. To simplify the modelling process by reducing the time and expertise needed.

2. To improve decision-making of the VLP tasks with realistic physical representations that are accessible for every stakeholders to be involved.

\section{Layout planning and modelling}

In the area of engineering research and practice, FLP is not a new problem. It has drawn wide attention from the manufacturing industry since the last century. FLP involves the allocation of various resources within the production environment that best address the requirements and constraints. A facility is an entity that facilitates the performance of any production task. It may be a machine tool, a work center, a manufacturing cell, a machine shop, a department, a warehouse, etc. [8]. It is understood that the placement of facilities in the factory would have substantial impact on many aspects of production. Decision on the alternative layouts are often determined through the comparison of defined features such as throughput, travel distance of material and operator, electrical power, interactions within manufacturing processes, aesthetics, safety, ergonomics, operator acceptance and etc. [9]. Therefore, FLP should be seen as a multi attribute decision making (MADM) task that need to meet both quantitative and qualitative measures.

Among the previous attempts of resolving FLP problems, algorithmic and procedural approaches are two major directions. Algorithmic approaches use mathematical modelling techniques to formulate the FLP as optimization problems and employ heuristic algorithms that simplify both design constrains and objectives to reach feasible solutions [6]. Quantitative measures such as flow distance of material and operators are the sole focus. Whereas procedural approaches can take both quantitative and qualitative measures in the design process [10]. It aims at dividing the design process into several steps which are solved sequentially [11]. However, the implementation heavily depends on the generation of quality design alternatives, which are largely based on experts' experience [12].

With the development of computer graphic technologies, VLP has drawn wide attention as the three-dimensional (3D) virtual models enable user experience that is closely related to the real world. It is reported that there are mainly three advantages for VLP [9]:

1. The ability to play "what if" and test alternative scenarios with relative ease;

2. Moving different actors who are affected by the layout design closer to the layout decision-making process;

3. Improve the decision making-process from both quantitative and qualitative perspectives given the richness and flexibility that virtual model can provide.

\section{Modeling virtual environment}

Among the various attempts of modeling the virtual environment, three major approaches can be identified based on how the virtual model is created. The first approach transforms existing physical facilities into virtual objects through devices such as camera or scanners. Methodologies and algorithms were developed to automatically extract and convert image, video data obtained into spatial data [13]. Another approach models facilities completely virtually in computers using computer-aided design (CAD) software or virtual reality modelling language (VRML) $[14,15]$. The third approach combines the previous two as a hybrid approach with the hope of benefiting from the advantages of both [16]. Each approach has its advantages and disadvantages. The camera and scanner approach is easy to perform and gets visually realistic model out of it. However, as the obtained model is static, it is difficult to implement interaction functions which is also one of the important aspects to the success of VLP. The CAD and VRML approach provides flexible possibilities to have different kinds of interactions, i.e. collision detection, but requires much higher expertise of the software tools and factory environments are usually too complicate to model in details. It is always a trade-off between the realistic level of the model and the time and expertise needed. The comparison of the three approaches are shown in the Table 1:
Table 1 The comparison of different virtual environment modelling approaches

\begin{tabular}{llll}
\hline Modeling tools & Model realistic & Model interactivity & Modelling complexity \\
\hline Camera and scanner & Medium, high & Low & Low \\
CAD and VRML & Low, medium & High & High \\
Hybrid & High & Medium, high & Medium \\
\hline
\end{tabular}


Table 2 The summary of three cases

\begin{tabular}{llllll}
\hline & Industry & Scale of change & Approach & VR types & Features \\
\hline Case I & Aerospace & Workstation & Hybrid & Desktop VR & Visualization \\
Case II & Trucks & Production cell & Hybrid & Immersive VR & Visualization, interaction \\
Case III & Snus and Tobacco & Packaging line & Hybrid & Immersive VR & Visualization, interaction \\
\hline
\end{tabular}

\subsection{Point cloud based virtual factory modelling}

In this section, we will explain the initial idea of the point cloud based virtual factory modelling. It is a hybrid approach that combines point cloud from 3D laser scanning and CAD models from conventional CAD software, to balance between model realistic, interactivity and complexity. 3D laser scanning is an active, non-contact, range measuring technology [17]. To capture the point cloud representation of the physical environment, the 3D laser scanner is positioned inside the area of interest and will emit the laser while capturing the returned reflection to measure the distance to the reflecting surface. Each scan takes approximately $4 \mathrm{~min}$ to capture the data. In a factory environment where machines and equipment are densely populated, the line of sight of the laser beam will be limited and the data capture needs to be repeated on several positions throughout the area in order to capture all the objects and surfaces. This results in multiple point cloud data sets which can be automatically registered into a common and coherent coordinate system to form the basis of the virtual factory model. When rendered on a computer screen, the point cloud represents a photorealistic 3D environment of the factory in scale 1:1 [18]. In a typical factory layout planning scenario, it also involves the replacement or re-location of equipment. Thus, CAD data of the new equipment can be rendered together with the captured point cloud to form the essential part of the virtual factory model. Depends on the requirement of each FLP task, the needed interaction functions can then be programmed to make the model alive and interactive, so that FLP tasks can be performed in the virtual environment.

\section{Virtual reality systems}

There are different ways of visualizing and interacting with the virtual models, based on the sense of presence [19], virtual reality (VR) systems can also be categorized them as:

- Desktop system.

- Wide-screen projection system.

- Immersive VR system using HMDs.

Desktop VR systems use monitors as the display device and conventional input devices such as mouse and keyboard for interaction. Wide-screen projection VR normally requires the setup of multiple projections screens with each screen covers a $20^{\circ}-30^{\circ}$ field of view (FOV). Thus to create a projection area over $100^{\circ}$ of $\mathrm{FOV}$, so that users experience a higher sense of presence in the virtual environment. Trackable gloves and controller are usually used as the input devices for interaction. Immersive VR systems offers a more flexible setup using the trackable HMD and controllers. Stereoscopic images of the virtual model are rendered in real time into the two displays in the HMD. The tracked movement of HMD is processed to live update the rendering of images so that users feel if they were present in the virtual model. The immersive VR systems have significant advantages as users feel as if they were present and interact with the real models. Thus potential design flaws are expected to be identified with relative ease in the design phase.

\section{Industrial cases}

Three case studies that adopted the point cloud based virtual factory modelling approach were conducted. The proposed approach is exemplified and refined through the cases. They are all industrial cases where layout changes were needed in existing production sites either for improving productivity or adaption of new products. The cases vary in the areas of manufacturing and the scale of the layout change.

Desktop VR was implemented in the first case and in the later cases, the immersive VR system using HMDs was chosen as the technology has becoming ever mature with much lower price and improved performance. With the $110^{\circ} \mathrm{FOV}$ and 90 frame per seconds (FPS) image rendering, it enables nature visualization that users feel as if they were present in the real environment and intuitive interactions within the virtual model. The cases are summarized in the Table 2.

\subsection{Case I}

\subsubsection{Background}

Aerospace components are rigidly controlled in both design and manufacturing. Each single component is tracked from cradle to grave to establish accountability and transparency in case of accidents. A new product or order often initiate the purchase of new equipment dedicated to the task. 
The company in this study had received a long term order that would guarantee steady demand for up to thirty years. With this in mind they were in the process of purchasing and installing twenty machines that were essentially going to be fixed for the duration of the supplier agreement. Any inefficiencies in the organization of the production system would remain so, bearing expensive relocations of the equipment. In order to thoroughly vet the installation plans before actually installing anything the company wanted to involve as many stakeholder perspectives as possible.

\subsubsection{Implementation}

3D laser scanning was conducted in the area allocated for installation of the new machines. Both a terrestrial laser scanner and a structured light hand held scanner was used [20]. Parts of the area contained existing, running, production equipment that had been marked for removal. The equipment ranged from material storage, to manual workstations, measurement tools, and CNC machines. The captured spatial data was processed and the equipment marked for removal was digitally extracted from the data set. The resulting point cloud was combined with CAD data of the purchased equipment to model in 3D the planned future situation. To enable a larger area in the VR model, some of the features of the model were converted into texturized meshes. Compared to colorized point cloud data size a reduction of up to $1000 \times$ can be achieved while sustaining comparable visual properties. Best suited for such a reduction are flat or close to flat features of the model, for example floor and wall areas. (Ref to WSC article for method)

\subsubsection{Evaluation}

In this case, the desktop/projector VR solution was chosen to visualize the virtual environment with the aim of facilitating workshop discussion regarding the layout change. The CAD model of each new CNC machine was placed according to a preliminary plan which relied heavily on the placement of existing foundations but didn't regard material flows or manual work tasks during the operation of the production. Additional equipment, such as material and tool carts, fixture stations, and information screens were added and positioned in the virtual model. The resulting model was combined with CAD data of the purchased equipment to model in 3D the planned future situation. It is visualized through 2D displays such as a computer monitor or a projector. Mouse and keyboard are used to control the movement within the virtual model.

The model was used during workshops with the project organization at the company. Stakeholders were able to explore and interact with the model to answer some basic qualitative questions. For example:
- Where should the information screen be positioned?

- Can you adequately reach the work space with the material handling cart?

- Are the fixture stations positioned in a good way?

The discussion results were then feed back into improve the layout change plan until a final consensus is reached. At the end of the workshop, they were also asked to evaluate their experience regarding the proposed approach for FLP by filling out a questionnaire.

\subsection{Case II}

\subsubsection{Background}

A Volvo truck manufacturing plant in United States was selected as it was facing challenges in the alignment of master process across its global plants. The firewall production cell has become one of the most evident bottleneck to cope with the increasing product variants and production flexibility. New equipment and production flows are needed to upgrade the current firewall production, thus a new production layout need to be designed and evaluated before implementation.

\subsubsection{Implementation}

3D laser scan was used to obtain the point cloud representation of the factory and resulted in a total of 82 individual scans, covering a large portion of the main assembly line in the plant [21]. The firewall subassembly production cell was selected as the focus for the case. Its core components were mainly captured in five scans, but data from surrounding areas were also included in the visualization to provide context to the cell as a part of the whole production system. CAD models of new resources were gathered and included in the virtual environment together with the point cloud data. In the integrated virtual environment, it consists of four different types of data sources: static point cloud as the background, meshed point cloud which are the walls and floor, interactive point cloud which represents two affected machines and interactive CAD model which is the new equipment. Immersive VR headset and motion tracking controllers are used to support visualization, navigation and interactions such as objects movement and feedback within the virtual model.

\subsubsection{Evaluation}

Nine participants from different actor groups within Volvo and one senior researcher in the field of virtual production from the research team at Chalmers took part in the evaluation. They were guided through a short training scenario and then presented with the VR model of the factory as shown in Fig 1 . They were asked to finish the following open tasks: 


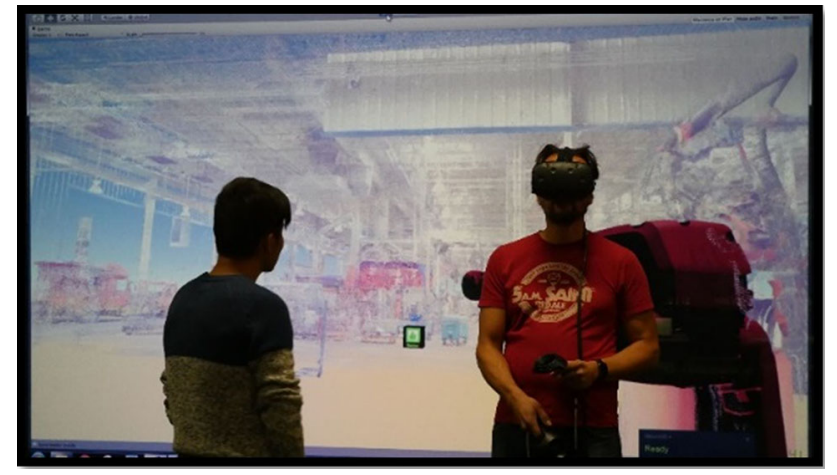

Fig. 1 Participant is exploring in the virtual factory to evaluate the layout

- Navigate in the 3D point cloud virtual factory.

- Modify the layout.

- Save and load new layout.

- Give feedback on the presented layout in the system based on their expertise.

The feedback is gathered for further improvement of the planned layout change. Questionnaire of both open-ended questions and close-ended scale ratings were used afterwards to collect their feedback regarding effectiveness of this proposed approach.

\subsection{Case III}

\subsubsection{Background}

A production line at the Swedish Match factory in Gothenburg is facing a reconstruction to reduce scrap due to varying product weight. New scales and surrounding equipment will be installed and will affect the production area which is already limited today. To ensure that access to the machines are sufficient and the operators can work in ergonomic postures, the production layout needs to be thoroughly planned and evaluated.

\subsubsection{Implementation}

Similar to the previous two cases, both point cloud data of the factory and CAD models of new facilities were gathered for the virtual factory modelling. Point cloud density was reduced in the surrounding area to balance between the realistic level of the model and the computing power needed. Machine sounds were recorded at the site and were set at the corresponding locations in the virtual model as spatial sounds to provide more immersive virtual experience. The integration process for this case involves three different data sources: static point cloud as the background, interactive CAD models of the new packaging line, and spatial sound sources that

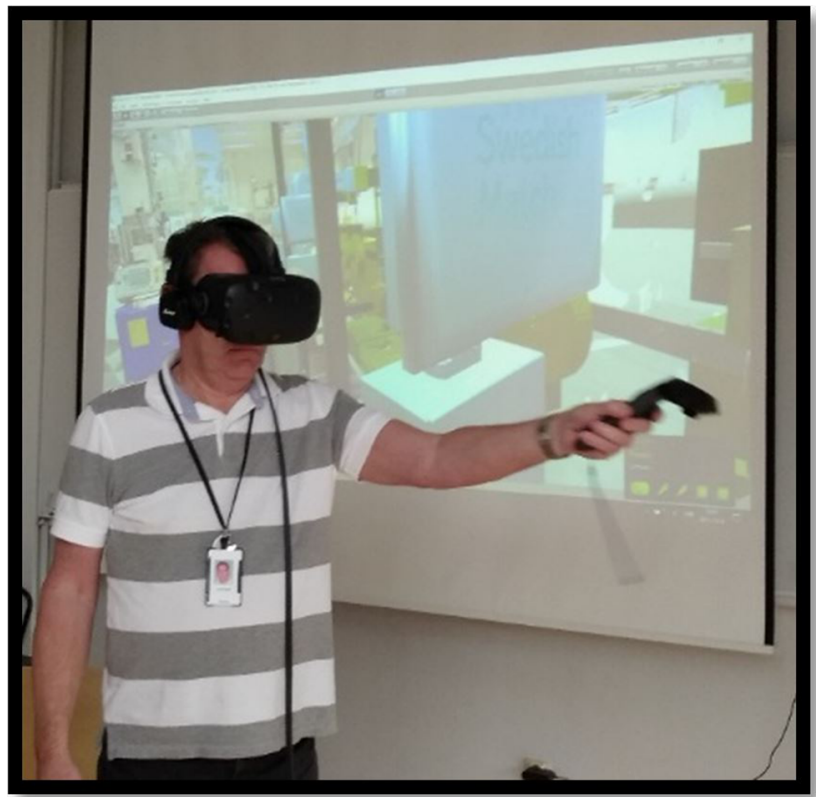

Fig. 2 Participant is exploring in the virtual factory to evaluate the accessibility of new layout

simulate the noise level. The old packaging line were removed from the point cloud data and replaced with the CAD model of the new line. The information screens of the new line were built as interactive objects which can be moved with the controller as a door hinge. Immersive VR headset and motion tracking controllers are used to support the visualization and interactions in the virtual model. It allows stakeholders to experience the planned setup and assess the virtual model, thus to stimulate constructive feedback regarding the planned layout.

\subsubsection{Evaluation}

Over 40 participants joined the evaluation workshop and assessed the planned layout in the virtual model using the immersive HMD and trackable controllers as shown in Fig. 2.

The participants are from various groups ranging from operator, project manager, maintenance engineer, manufacturing engineer, board member, etc. which covers the full list of stakeholders in the company. Each participant were guided with a short introduction to the VR device. Then they were asked to navigate and observe the planned change in the virtual model and perform the accessibility assessment. The feedback on the proposed layout was collected and used as the basis for the plan improvement. At the end, a questionnaire with scale ratings and open questions was handed out to collect their feedback and 30 participants responded to the questionnaire. 


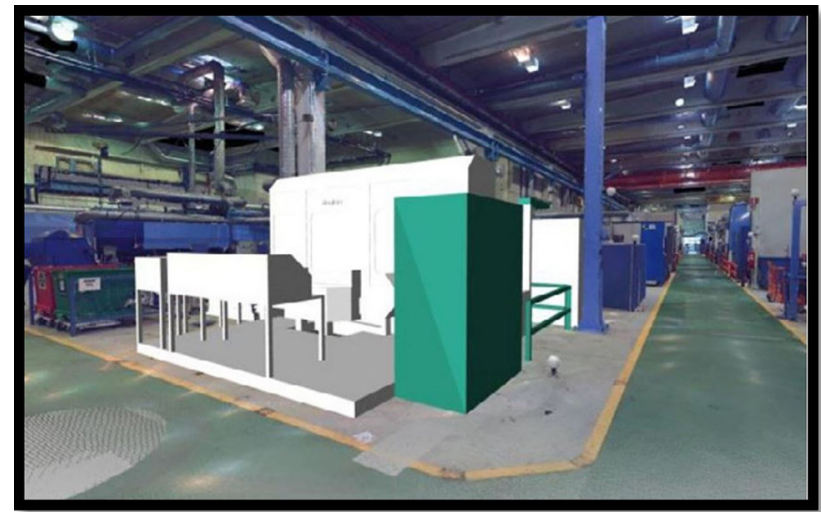

Fig. 3 Participant is exploring in the virtual factory to evaluate the accessibility of new layout

\section{Results}

The results are divided into two parts: first, the results of the three cases are analyzed and summarized. Then a general guidance is extracted for future implementation of point cloud based virtual factory modelling in FLP tasks.

\subsection{Cases results}

\subsubsection{Case I}

The point cloud data and CAD models were integrated to build the hybrid virtual factory model, shown in Fig. 3. The model is accessible through a desktop computer and a projector was used to facilitate group discussion during the workshop.

Project members were impressed by the visualization possibility that the point cloud based virtual factory model holds and found possible areas of application for their specific interest. All participants realized that the machine and supporting equipment require more space by analyzing the hybrid model. With the first proposed layout it would not have been possible to store enough trolleys with material and products close to the machine. With this new information at hand, the group discussed alternative solutions, which were then tested in the hybrid model. 3D CAD objects and point cloud objects were translated in the model to the suggested positions by the group, ultimately resulting in a new layout proposal. Currently, the model requires an expert user to control for navigation and modification. However, it could be discussed whether this should be necessary or not. If the virtual model were made to be accessible by every stakeholders, it may improve the outcome of the discussion even further.

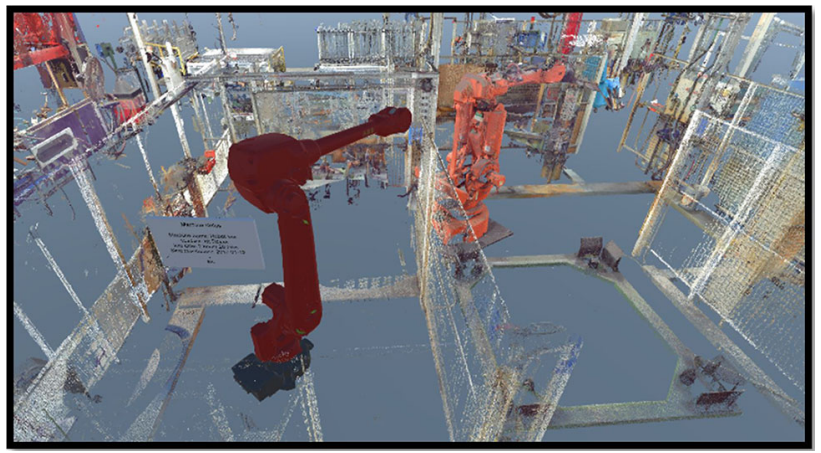

Fig. 4 Participant is exploring in the virtual factory to evaluate the accessibility of new layout

\subsubsection{Case II}

A virtual factory model was developed based on the captured point cloud data and CAD models of planned equipment, as shown in Fig. 4. It is accessible by stakeholders through an immersive HMD and controllers to navigate around the virtual factory, pick and place interactive objects to modify layouts, save and load layouts for peer review, and leave feedback within the virtual model.

\subsubsection{Case III}

A virtual factory model was created based on the planned layout change as shown in Fig. 5. It consists of both point cloud data and CAD models, while interaction functions such as navigation, drag and move were implemented to enable the general evaluation of the virtual model and the accessibility assessment of the workstation at the new line.

\subsubsection{Statistical analysis from case II and case III}

As part of the evaluation in case 2 and case 3, four statements were ranked using scale analysis, using a four-level Likert scale (strongly disagree, disagree, agree, and strongly agree), illustrated in Table 3. A total of 49 persons were participating in the workshops. It is worth noting that there are 9 participants who did not fill in the questionnaire due to their time limit or other reasons. As a result, some critiques might be hidden behind the scene. For the correlation statistics 32 answers were valid. The reliability statistics (Cronbach alpha) was $0.875(\mathrm{~N}=4)$ for this study.

The mean value was high for all statements, illustrated in Table 4, which indicates that this technology is seen as a mature technology that is easy to use. Hence, the attendees had hard time to see where it could be useful in their daily work. The majority of the test subjects in case II were positive with the potential benefits of this VR approach and would like to share or recommend the system for a wide usage. Due to 
(a)

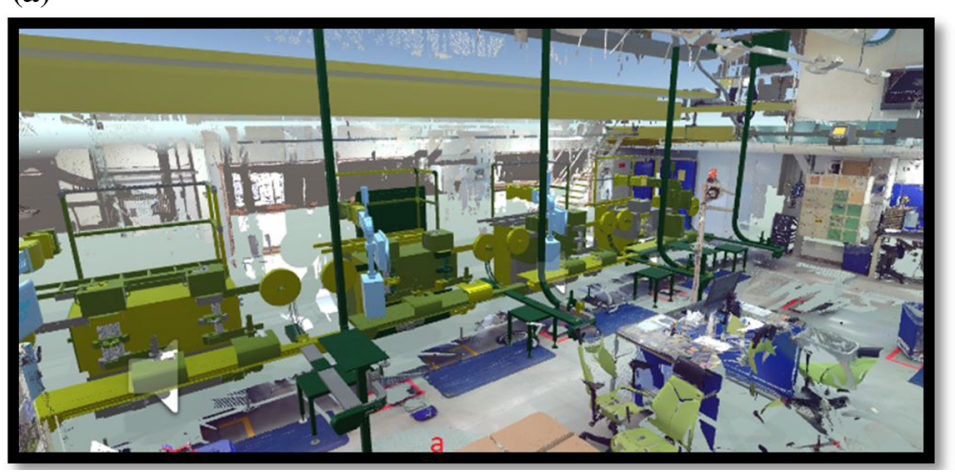

(b)

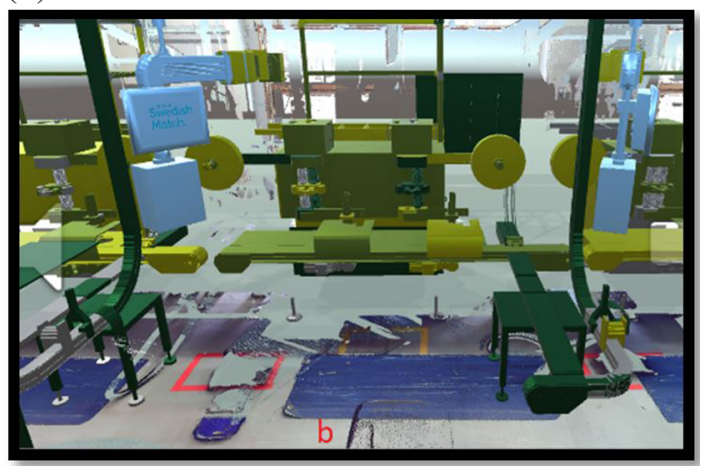

Fig. 5 Overview (a) and planned workstation (b) in virtual factory model

Table 3 Descriptive statistics of the survey results

\begin{tabular}{llll}
\hline Statements & Means & SD & N \\
\hline Easy to use & 3.45 & 0.597 & 40 \\
Clear benefit & 3.55 & 0.552 & 40 \\
Useful to my job & 3.16 & 0.628 & 32 \\
Recommend to others & 3.58 & 0.502 & 33 \\
\hline
\end{tabular}

the fact that the tool is in prototype phase of the concept, user experience related ratings were not as good as the potential benefits. The correlation between the statements shows the same pattern as the mean value, illustrated in Table 4.

The strongest and significant correlation is between easy to use and recommend (0.939), which means that the attendees thought that is was easy to use and understand the tools, but also that they would recommend the tools to others. Positive benefits was also the visually representative of the real factory, accurate and "near" life like experience. They could also see a clear benefit with the tools and the easiness of using the tool (0.863). There were moderate or weak correlation between 'useful to my job' and the other statements. This could depend on lack of business models, and maturity within the organizations on using these tools in the daily job.
We believe that this will increase over the next few years due to the fast development and improvement of technology, but also increase of use within companies. When analyzing the open-end questionnaire feedback, some recurring themes are identified such as easy to navigate around and visualize the planned layout, effectively creates the basis for improved discussion around the planned layout among stakeholders. The immersive visualization and interaction in the virtual model enable stakeholders from different backgrounds to gain a coherent understanding about the planned change. From the layout planning perspective, it helps all stakeholders to be actively involved in the planning process, which will not only reduce potential design flaws but also improve user acceptance when new layout is implemented. At the same time, some obstacles were detected; dizziness while using the HMD and disorientation in the virtual environment. Additionally, two test subjects believed that the tool as such is different from what they are used to, thus it takes time to learn and get familiar with. Three major challenges were noted as data compatibility, organizational attitudes, and cost. Data of the various aspects of the production system resides in many internal systems and in different formats. It is still a cumbersome process to integrate various data sources into the virtual environment.

Table 4 Correlations between the different statements

\begin{tabular}{llllll}
\hline & & Easy to use & Clear benefit & Useful to my job & Recommend \\
\hline Easy to use & Pearson correlation & 1 & $0.863^{\mathrm{a}}$ & $0.509^{\mathrm{a}}$ & 0.003 \\
& Sig. (2-tailed) & - & 0.000 & $0.496^{\mathrm{a}}$ & 0.000 \\
Clear benefit & Pearson correlation & $0.863^{\mathrm{a}}$ & 1 & 0.004 & $0.768^{\mathrm{a}}$ \\
& Sig. (2-tailed) & 0.000 & - & 1 & 0.000 \\
Useful to my job & Pearson correlation & $0.509^{\mathrm{a}}$ & $0.496^{\mathrm{a}}$ & - & $0.518^{\mathrm{a}}$ \\
\multirow{2}{*}{ Recommend } & Sig. (2-tailed) & 0.003 & 0.004 & $0.518^{\mathrm{a}}$ & 0.002 \\
& Pearson correlation & $0.939^{\mathrm{a}}$ & $0.768^{\mathrm{a}}$ & 0.002 & - \\
\hline
\end{tabular}

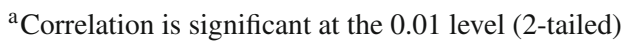




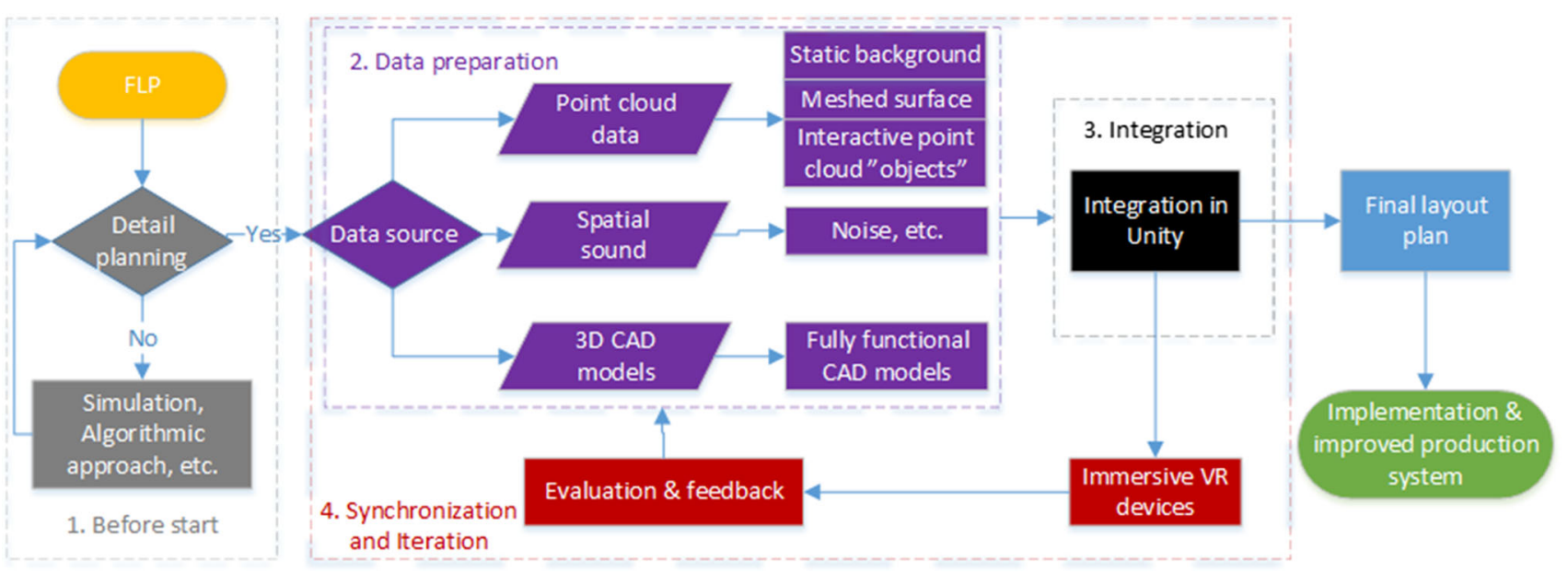

Fig. 6 Conceptual framework of the guidance to hybrid virtual factory modelling

\subsection{Guidelines for point cloud based virtual factory modelling}

With the three cases described above, the hybrid approach that combines point cloud data with 3D CAD models to build virtual factory for layout planning were demonstrated and tested. The results show that it is a promising path towards efficient and effective decision support in (re)designing factory layout with the following advantages:

- Fast modelling process of the virtual factory;

- Realistic virtual representation and interaction that facilitate qualitative feedback;

- Easy modification of layout design,

- Collaborative environment that could involve all stakeholders.

To streamline the point cloud based virtual factory modelling approach for future implementation to FLP tasks, a general guidance is extracted and shown in Fig. 6.

\subsubsection{Before start}

FLP problems vary in terms of the scope and the purpose of change. The point cloud based virtual factory modelling approach intends to support the detail layout planning with focus on gaining qualitative feedback regarding safety, accessibility, and ergonomics. It is especially suitable for upgrading existing factory due to the combinational characteristics of rapid capturing virtual representation of physical world and integration with 3D CAD models.

\subsubsection{Data preparation}

When the FLP task is assessed and considered appropriate to taking the proposed approach, the next step is to prepare different types of data to construct the virtual factory model. The virtual model created consists with 3 major types of data: point cloud, 3D CAD models and spatial sound. Based on the complexity of the post-processing procedures, the point cloud data can be further categorized as point cloud data sets that are non-interactive, meshed and interactive. Noninteractive ones are the raw data captured through 3D laser scanner, which needs the least effort of post-processing, but require high computation cost to visualize it. It can be used as the background in the virtual environment to provide contextual information. Meshed ones are 3D objects that are generated from point cloud data using algorithm. However, existing algorithms for point cloud objectification only work well with simple geometry or surface recognition. Therefore, it can be used for flat area such as the walls and floor to reduce the computation cost. Interactive point cloud data sets can be created by adding transparent bounding boxes that wrap the relevant point cloud into a group. It enables the interactions such drag and drop with relative low effort. For the facilities that need comprehensive behaviors, 3D CAD models need to be developed. Spatial sound such as equipment running noise would increase the level of presence, which is beneficial to ergonomic concerns. The different types of data are illustrated in Fig. 7.

As discussed in the previous section, it is a question of balancing modeling complexity and the model quality. Prior to the data collection, it is necessary to determine the level of details needed for each objects. Thereafter, data collection can be proceeded accordingly. 

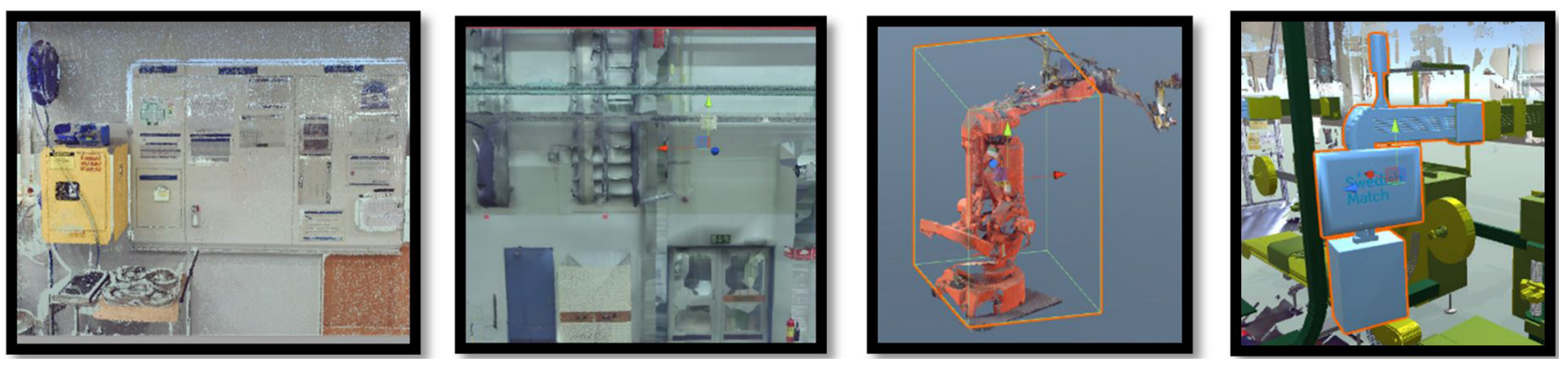

Fig. 7 Static, meshed and interactive point cloud, interactive CAD (from left to right)

Table 5 Implemented functions of each case

\begin{tabular}{lllc}
\hline Functions & Descriptions & Case I & Case II \\
\hline Visualization & Desktop screen or projector or HMD & $\times$ & $\times$ \\
Navigation & Mouse and keyboard or controller teleport through VRTK [23] & $\times$ & $\times$ \\
Pick and place object & Collision detection, controller button & $\times$ & $\times$ \\
Save and load layout & Serialization of object position and rotation & $\times$ \\
In-app feedback & Collision detection and UI & $\times$ \\
Accessibility & Collision detection, controller button, hands & & $\times$ \\
\hline
\end{tabular}

\subsubsection{Integration}

After the various data are prepared, they need to be integrated into one platform in order to create a single interface for uses to access it. There are many such platforms available and we chose to use Unity3D [22] which is a cross-platform game engine primarily used to develop video games and simulations for computers, consoles and mobile devices. The mature developer community and large number of working assets in this platform ensures the quick and quality integration process of the virtual factory model. The virtual factory model is completed by integrating data resources prepared in the previous steps with scripts to enable users to manipulate the model for the designing and evaluation of new factory layouts. Various interaction functions can be implemented based on the different requirements of the FLP tasks. In Table 5, it lists the functions that have been implemented for the three cases as a reference.

\subsubsection{Synchronization and iteration}

After the VFM is prepared, layout engineer can design and modify virtual layout for further evaluation and assessment through the involvement of all the stakeholders. The collaborative effort that involves every stakeholders in the designing process of new layout enables early detection of potential flaws and ensures the quality of the planned layout. The realistic visualization and intuitive interactions in the immersive environment facilitate the feedback process to be efficient and effective. In this way, the feedback is synchronized to modify the virtual layout and iterate until a final plan that is agreed with all stakeholders can be reached for implementation.

\section{Discussion}

The hybrid approach of combining point cloud data with 3D CAD models to build virtual factory for layout planning were demonstrated and tested. The results show that it is a promising path towards efficient and effective decision support in (re)designing factory layout with the following advantages:

While the studies have shown the significant benefits of using the point cloud based virtual factory modelling for layout planning. There are many challenges identified need to be resolved to ensure the transition from the conventional approaches to the virtual layout planning approach. In this section, the identified challenges are discussed as for the future research directions.

The first challenge is the post processing of point cloud data, or the objectification of point cloud. 3D laser scanner is capable of rapid capturing realistic virtual representation of the real world without much expert knowledge, the technology has certain constraints as well. First of all, the captured point cloud data needs some post-processing procedures such as objectification to make the data applicable for more application scenarios. Automatic objectification algorithm is still in the premature phase, while manual process can be tedious and time-consuming. Another challenge is to keep the point cloud data up-to-date, as production systems are not static. 
So there is the need to have the equipment and infrastructure in place to handle the continuous 3D scanning and updating of the point cloud data. At the same time, the increasing demand of higher computing power is also a hinder. This is especially important in the VR applications as any lag of image or lower frequency rate would make the user suffer from dizziness and other ergonomic issues.

Second is the CAD data compatibility, as another major data sources of building the virtual model is the 3D CAD models. Today, there are many different standards and formats for these models. Different companies have their own ritual or preference over the CAD software they are working with. To transform various models into VR ready ones requires tedious conversion process. Valuable information such as color, geometry attributes of the model might lost in the conversion process. Thus, individual workflow of data conversion is an unfavorable but needed process in today's work practice. The development of a universal standard of modelling 3D objects for VR applications would save much unnecessary process of converting in between different data sources.

The user interface (UI) design in the VR environment is another challenge. Previously there have been many research focused on UI design for desktop applications. However, the immersive VR has brought new ways of presenting information and interacting with the system, proven theories work in desktop applications might not fit well in the VR application. Users will need time to learn and get familiar with the new input and output methods. At the same time, further studies of standardized VR user interface and interaction design will help to ease the learning process. The questionnaire results of the four industrial cases also indicate most users experienced difficulty of using the HMD and controllers at the beginning of the tests and they noted that the interaction and UI need improvement.

Besides the technological challenges discussed above, the organizational attitudes and user acceptance towards the VR technologies are the key factors of successful adaption. This requires better communication and more training that introducing and incorporating VR into existing work methods as well as standardize VR interaction design.

\subsection{The interactive approach}

In VR systems, the interactive experience is fundamental to provide users the seamless experiences as she/he is in real world [24], which would heavily influencing the quality of FLP carried out through the proposed VR system. Therefore, it is important to provide interactivity features in the system for better user experience and quality of work result [25, 26]. As a result studies about the development of interactive virtual environment has attracted more attention [27].
In this study, the interactive nature of the proposed guidelines is manifested in two folds: the interactivity of the VR systems, and the interactivity of the FLP process. Different interaction designs have been implemented and tested out in the VR system to make the experience closer to reality. For example, the spatial sound which responses in real-time to user position change, has helped increased the level of presence in the virtual environment and ultimately improve the FLP quality. At the same time, the iterative process of refining layout plans through the VR system described in the guidelines make it possible to get all the affected stakeholders to be actively involved and contribute in the FLP process.

\section{Conclusion}

In this paper, we proposed a point cloud based virtual factory modelling approach for the FLP tasks. The proposed approach was exemplified and refined through three industrial cases. The case results show that the point cloud based virtual factory modelling approach can create realistic virtual models for the FLP tasks. With the immersive HMD and trackable controllers, one can easily interact with the virtual model to perform FLP tasks. The realistic virtual model and nature interaction lowered the required expertise which enables stakeholders with different background to be actively involved and contribute to the new layout design. It is arguable whether the proposed approach simplifies the modelling process of virtual factories, as the limitations discussed in the previous section, especially, the post-processing of point cloud data is still cumbersome. However, with the development of machine learning in the field, it can be improved in the near future.

For further improvement, research can be conducted in areas such as point cloud data objectification using machine learning, 3D data compatibility, user-centered design of industrial VR applications.

Acknowledgements Funding was provided by VINNOVA.

Open Access This article is distributed under the terms of the Creative Commons Attribution 4.0 International License (http://creativecomm ons.org/licenses/by/4.0/), which permits unrestricted use, distribution, and reproduction in any medium, provided you give appropriate credit to the original author(s) and the source, provide a link to the Creative Commons license, and indicate if changes were made.

\section{References}

1. Tompkins, J.A., White, J.A., Bozer, Y.A., Tanchoco, J.M.A.: Facilities Planning, 3rd edn. Wiley, Hoboken (2003)

2. Muther, R.: Systematic Layout Planning, 2nd edn. Cahners Books, Boston (1974) 
3. Sly, D.P.: Systematic approach to factory layout and design with FactoryPLAN, FactOPT, and FactoryFLOW. In: Proceedings of the Winter Simulation Conference, pp. 584-587 (1996)

4. Grajo, E.S.: Strategic layout planning and simulation for lean manufacturing. In: Proceedings of the Winter Simulation Conference, pp. 564-568 (1996)

5. Jiang, S., Nee, A.Y.C.: A novel facility layout planning and optimization methodology. CIRP, Ann. -Manuf. Technol. 62(1), 483-486 (2013)

6. Jiang, S., Ong, S.K., Nee, A.Y.C.: An AR-based hybrid approach for facility layout planning and evaluation for existing shop floors. Int. J. Adv. Manuf. Technol. 72(1-4), 457-473 (2014)

7. Yaman, R.: Establishment and use of virtual layouts for manufacturing. Integr. Manuf. Syst. 12(6), 400-408 (2001)

8. Heragu, S.S.: Facility Design, 4th edn. CRC Press, Boston (2016)

9. Smith, R.P., Heim, J.A.: Virtual facility layout design: the value of an interactive three-dimensional representation. Int. J. Prod. Res. 37, 3941 (1999)

10. Yang, T., Kuo, C.: A hierarchical AHP/DEA methodology for the facilities layout design problem. Eur. J. Oper. Res. 147, 128-136 (2003)

11. Mahdavi, I., Shirazi, B., Paydar, M.M.: A flow matrix-based heuristic algorithm for cell formation and layout design in cellular manufacturing system. Int. J. Adv. Manuf. Technol. 39(9), 943953 (2008)

12. Shahin, A., Poormostafa, M.: Facility layout simulation and optimization: an integration of advanced quality and decision making tools and techniques. Mod. Appl. Sci. 5(4), 95-111 (2011)

13. Zetu, D.A.N., Banerjee, P., Schneider, P., Zetu, D.A.N., Banerjee, P., Schneider, P.: Data input model for virtual reality-aided facility layout data input model for virtual reality-aided facility layout. IEE Trans. 30, 597-620 (1998)

14. Duffy, V.G., Wu, F.F., Ng, P.P.W.: Development of an Internet virtual layout system for improving workplace safety. Comput. Ind. 50(2), 207-230 (2003)

15. Deshmukh, P., Valles-Rosales, D.J.: Virtual factory approach to optimize an assembly process. In: IIE Annual Conference and Exposition (2005)

16. Luo, Y.B., Ong, S.K., Chen, D.F., Nee, A.Y.: An Internet-enabled image- and model-based virtual machining system. Int. J. Prod. Res. 40(10), 2269-2288 (2002)

17. Beraldin, J.-A., Blais, F., El-Hakim, S., Cournoyer, L., Picard, M.: Traceable 3D imaging metrology: evaluation of 3D digitizing techniques in a dedicated metrology laboratory. In: Proceedings of the 8th Conference on Optical 3-D 3D measurement Techniques on Territory Laser Scanning I, pp. 310-318 (2007)
18. Erik. Lindskog, Towards realistic visualisation of production systems. Chalmers University of Technology (2004). Retrieved from https://research.chalmers.se/en/publication/198091

19. Korves, B., Loftus, M.: The application of immersive virtual reality for layout planning of manufacturing cells. Proc. Inst. Mech. Eng. Part B J. Eng. Manuf. 213, 87-91 (1999)

20. Lindskog, E., Berglund, J., Vallhagen, J., Johansson, B.: Visualization support for virtual redesign of manufacturing systems. Proc. CIRP 7, 419-424 (2013)

21. Gong, L., Berglund, J., Saluäär, D., Johansson, B.: A novel VR tool for collaborative planning of manufacturing process change using point cloud data. Proc. CIRP 63(C), 336-341 (2017)

22. UnityTechnology: What is unity? 2017. [Online]. https://unity3d. com/unity

23. VRTK: Virtual reality toolkit: a productive VR toolkit for rapidly building VR solutions in Unity3d. (2017). [Online]. https:// vrtoolkit.readme.io/. Accessed 20 Nov 2017

24. Vergnano, A., Berselli, G., Pellicciari, M.: Interactive simulationbased-training tools for manufacturing systems operators: an industrial case study. Int. J. Inter. Des. Manuf. 11, 785-797 (2017)

25. Elzer, P., Weisang, C., Zinser, K.: Knowledge-based system support for operator tasks in S\&C environments. In: Proceedings of IEEE International Conference on Systems, Man and Cybernetics, Cambridge, MA, USA, vol. 3, pp. 1078-1083 (1989)

26. Dauphinee, J.L.: Development and implementation of a sustainable operator training and certification process. In: Proceedings of IEEE International Semiconductor Manufacturing Symposium, San Jose, CA, USA, pp. 509-512 (2001)

27. Valentini, P.P.: Enhancing User Role in Augmented Reality Interactive Simulations. Human Factors in Augmented Reality Environments. Springer, New York (2013)

Publisher's Note Springer Nature remains neutral with regard to jurisdictional claims in published maps and institutional affiliations. 